\title{
Extraction and Analysis of Chaotic Characteristics about Multiple Physiological Signals under Different Emotions Nie Chun yan ${ }^{1, a}$, Wang Ju $u^{1, b}$, He Fang ${ }^{2, c}$, Sato Reika ${ }^{3, d}$ \\ ${ }^{1}$ Electronic Information Institute Changchun University, China \\ ${ }^{2}$ School of Electronics and Information Engineering, Changchun University of Science and Technology, Changchun 130022, China \\ ${ }^{3}$ School of Design, Jiangnan University, 1800 Lihu Dadao BinHu Qu, Wuxi,China \\ ae-mail: ncy66@163.com, be-mail: youyou8221@yahoo.com.cn, \\ ce-mail: hefang0724@sina.cn, ${ }^{\mathrm{d}}$ e-mail:satoreika@dg.osakac.ac.jp
}

Keywords: multiple physiological signals, emotion recognition, chaos

\begin{abstract}
We extract the chaotic characteristics of multiple physiological signals in this paper, and the relevant algorithms for extracting chaotic characteristics are given respectively. Based on different algorithms we respectively calculate four kinds of chaotic characteristic parameters from multiple physiological signals, such as maximum Lyapunov exponent, the correlation dimension, approximate entropy and complexity, to make them connect with the connotation of physiological signals not through the emotions. If we establish chaotic characteristic matrix by the extracted chaotic characteristic parameters, use the classifier to map the chaotic characteristics to a given category, so the problem of emotion analysis based on the multiple physiological signals is converted to the problem of classification in pattern recognition, which lay a foundation for better emotion recognition.
\end{abstract}

\section{Introduction}

In the nineties of the last century, chaos theory began to apply in the field of biology and medical, Goldberger[1] put forward the conclusion that the dynamic characteristics of normal, healthy life system is "chaotic". Physiological signal are the extrinsic manifestation of emotions, that is to say the changes of emotions can express by the changes of physiological signals. This performance is not controlled by the individual's subjectivity, so the result is more objective and real. Chaotic parameter characteristics are different from traditional statistical characteristics, which can reflect the nonlinear properties of physiological signals essentially. We can obtain some statistical characteristics of physiological signals namely the chaotic characteristic parameters of physiological signals by chaos theory. Those chaotic characteristic parameters provide a new method for the internal mechanism research and emotion analysis of physiological signals. Therefore, in this paper, through the chaotic characteristic parameters that we extracted from physiological signals, lay a foundation for following emotion recognition. In the analysis of physiological signals, some nonlinear method is used to analysis the physiological signals, but they tend to analysis one single physiological signal, while in this article we extract the chaotic characteristics of multiple physiological signals, using different algorithms to respectively calculate four kinds of chaotic characteristic parameters from multiple physiological signals. In this article, we extract the chaotic characteristic parameters of multiple physiological signals (ECG, EMG, RSP, SC) under four different emotions (joy, anger, sadness, pleasure). such as maximum Lyapunov exponent, the correlation dimension, approximate entropy and complexity.

\section{The Algorithms for Extracting Chaos Characteristic Parameters}

\section{A. Calculate of the maximum Lyapunov exponent and analysis}

Now according to the definition we can calculate the Lyapunov exponent. In addition, there are many other methods to calculate the Lyapunov exponent, the methods are divided into two main 
categories: the method of Wolf[2] and Jocobian[3]. Wolf method is applicable to the sequence without noise, Jocobian method is suitable for the sequence with large noise. For calculating the maximum Lyapunov exponent of time sequence, this paper uses the classic Wolf method.

According to the Wolf[4] method we can calculate the maximum Lyapunov exponent of EMG, SC and RSP signals under four different kinds of emotions, their values range are shown in table 1.

Table 1 The maximum Lyapunovexponent of EMG, SC and RSP signals under four different emotions

\begin{tabular}{cccccc}
\hline & samples & ECG & EMG & RSP & SC \\
\hline Joy & 25 & $0.0104 \sim 0.0704$ & $0.1688 \sim 1.5614$ & $0.0085 \sim 0.0782$ & $0.0171 \sim 0.1563$ \\
Anger & 25 & $0.0143 \sim 0.1022$ & $0.0991 \sim 0.9338$ & $0.0213 \sim 0.0892$ & $0.0915 \sim 0.2094$ \\
Sadness & 25 & $0.0042 \sim 0.1762$ & $0.0116 \sim 0.6137$ & $0.0087 \sim 0.1164$ & $0.1637 \sim 0.2155$ \\
Pleasure & 25 & $0.0136 \sim 0.1021$ & $0.0697 \sim 0.8860$ & $0.0171 \sim 0.1006$ & $0.0112 \sim 0.1782$ \\
\hline
\end{tabular}

In the table 1, according to the values range of maximum Lyapunov exponent, we can distinguish emotions Joy and Sadness, but the values range of maximum Lyapunov exponent of the other two emotions Anger and Pleasure have some overlap. Similarly, three physiological signals ECG, EMG and RSP, their maximum Lyapunov exponent range also have a certain overlap under four different kinds of emotions, so we can draw that it is not simple to distinguish different emotion states through maximum Lyapunov exponent. Therefore, in order to distinguish the four different kinds of emotions, we need to find out the combination of different chaotic characteristic parameters further more.

B. Calculate of The Correlation Dimension and Analysis

Correlation dimension is a invariant that describe the self-similar structure of attractors, it is equally important as the maximum Lyapunov exponent in chaotic characteristic parameters, the common method to calculate the correlation dimension is the G-P algorithm[5].

According to the G-P algorithm we can also calculate the correlation dimension of EMG, SC and RSP signals under four different kinds of emotions, their values range are shown in table 2.

Table 2 The correlation dimension of EMG, SC and RSP signals under four different emotions

\begin{tabular}{cccccc}
\hline & samples & ECG & EMG & RSP & SC \\
\hline Joy & 25 & $0.7381 \sim 1.8733$ & $0.1594 \sim 3.8442$ & $0.0085 \sim 0.0782$ & $1.9685 \sim 2.8717$ \\
Anger & 25 & $0.7176 \sim 2.2939$ & $0.6844 \sim 5.0392$ & $0.0213 \sim 0.0892$ & $1.9004 \sim 3.6804$ \\
Sadness & 25 & $0.5243 \sim 1.9127$ & $0.2121 \sim 3.7841$ & $0.0087 \sim 0.1164$ & $1.7162 \sim 2.8991$ \\
Pleasure & 25 & $0.5544 \sim 2.2734$ & $0.1982 \sim 4.9567$ & $0.0171 \sim 0.1006$ & $1.8015 \sim 2.6637$ \\
\hline
\end{tabular}

In the table 2, under the emotions of Joy and Pleasure, their values range of correlation dimension are similar, we can not tell the differences between the two emotions use our naked eye. Similarly, three physiological signals ECG, EMG and RSP, the range of their correlation dimension also have some overlap under four different kinds of emotions.

C. Calculate of The Approximate Entropy and Analysis

Approximate entropy can show the internal complexity of the signals, its calculating algorithm is simple and has strong anti-interference ability. In this section, the author will calculate the approximate entropy of physiological signals under different kinds of emotions according to the definition of approximate entropy[6].

According to the definition of approximate entropy we can also calculate the approximate entropy of EMG, SC and RSP signals under four different kinds of emotions, their values range are shown in table 3.

Table 3 The approximate entropy of EMG, SC and RSP signals under four different kinds of emotions

\begin{tabular}{cccccc} 
& samples & ECG & EMG & RSP & SC \\
\hline Joy & 25 & $0.1452 \sim 0.6668$ & $0.3765 \sim 1.7244$ & $0.1542 \sim 0.4654$ & $0.0214 \sim 0.5026$ \\
Anger & 25 & $0.1416 \sim 0.5373$ & $0.1983 \sim 1.8323$ & $0.3433 \sim 0.4397$ & $0.0643 \sim 0.3497$ \\
Sadness & 25 & $0.1317 \sim 0.6063$ & $0.1432 \sim 1.2685$ & $0.1691 \sim 0.1916$ & $0.0565 \sim 0.3249$ \\
\hline
\end{tabular}




$\begin{array}{llllll}\text { Pleasure } & 25 & 0.1224 \sim 0.4628 & 0.3578 \sim 1.3317 & 0.2272 \sim 0.2883 & 0.0292 \sim 0.4714\end{array}$

In the table 3, compared with the values range of approximate entropy of the other three kinds of emotions, the value range of approximate entropy is larger under the emotion of Joy, while the other three kinds of emotions can be obviously distinguished by the values range. Similarly, three physiological signals ECG, EMG and RSP, the range of their approximate entropy also have a certain overlap under four different kinds of emotions, that is to say the differentiation of the approximate entropy to different emotions is not high.

D. Calculate of The Complexity and Analysis

Approximate entropy can describe the complexity of the signals, the definition of the entropy, however, is too theoretical, so in order to describe the actual sense of the complexity, we introduce another nonlinear characteristics - complexity[7].

According to the definition of complexity we can also calculate the complexity of EMG, SC and RSP signals under four different kinds of emotions, their values range are shown in table 4.

Table 4 The complexity of EMG, SC and RSP signals under four different emotions

\begin{tabular}{cccccc}
\hline & samples & ECG & EMG & RSP & SC \\
\hline Joy & 25 & $0.1002 \sim 0.2658$ & $0.2504 \sim 0.9047$ & $0.0925 \sim 0.1655$ & $0.0115 \sim 0.2966$ \\
Anger & 25 & $0.1154 \sim 0.2155$ & $0.1846 \sim 0.7586$ & $0.1734 \sim 0.2273$ & $0.0194 \sim 0.4044$ \\
Sadness & 25 & $0.2194 \sim 0.2311$ & $0.1306 \sim 0.6931$ & $0.1041 \sim 0.1347$ & $0.0195 \sim 0.2234$ \\
Pleasure & 25 & $0.1077 \sim 0.1811$ & $0.2465 \sim 0.5545$ & $0.1041 \sim 0.1308$ & $0.0115 \sim 0.2348$
\end{tabular}

In the table 4, we can distinguish the two emotions between Joy and Anger according to the values range of complexity, while the emotions of Sadness and Pleasure, their values range of complexity are basically overlapped, thus we can only distinguish the two emotions between Joy and Anger by our naked eye. Similarly, through the observation three physiological signals ECG, EMG and RSP, the range of their complexity also have a certain overlap under four different kinds of emotions, so we can draw that it is not simple to distinguish different emotional states through complexity.

In conclusion, calculate the maximum Lyapunov exponent, the correlation dimension, the approximate entropy and the complexity of each sample, we can establish characteristic matrix of the samples, which lay a foundation for the analysis of emotions.

\section{The Comprehensive Analysis between The Chaotic Characteristics and Associated Emotions}

Use different algorithms to extract four chaotic characteristic parameters, which are respectively the maximum Lyapunov exponent, the correlation dimension, the approximate entropy and the complexity. So for four kinds of physiological signals, we get a total of 16 chaotic characteristic parameters, they are respectively the maximum Lyapunov exponent, the correlation dimension, the approximate entropy and the complexity of ECG signal, EMG signal, RSP signal and SC signal.

According to the values range of the 16 chaotic characteristic parameters we preliminarily get the conclusion that the values range of maximum Lyapunov exponent of SC signal can distinguish the emotions between Joy and Sadness, but with the other two kinds of emotions, their values range of maximum Lyapunov exponent have some overlap. Under the emotions of Joy and Pleasure of SC signal, their values range of correlation dimension are similar, we can not tell the differences between the two emotions use our naked eye. The approximate entropy values range of RSP signal, under the emotion of Joy the value range of approximate entropy is larger, while the other three kinds of emotions can be obviously distinguished by their values range. According to the value range of complexity of RSP signal we can distinguish the emotions between Joy and Anger, while the emotions of Sadness and Pleasure, their values range of complexity are basically overlapped, thus we can only distinguish the two emotions between Joy and Anger by our naked eye. 


\section{Conclusion}

Based on the above analysis, the value ranges of single chaotic characteristic parameter have a certain overlap, so we can't clearly distinguish the four different kinds of emotions. Therefore, in order to distinguish the four different kinds of emotions, we need to find out the combination of different chaotic characteristic parameters further more. Based on chaos theory of multiple physiological signals, use the classifier to map the chaotic characteristic to a given category, so the problem of emotion analysis is converted to the problem of classification in pattern recognition.

\section{Acknowledgment}

This work is partially supported by The Ministry of education "chunhui plan" Grant No. Z2014136 and Jilin Province Science Foundation Grant No. 201215110.

\section{References}

[1] Goldberger AL. Non-linear dynamics for clinicians: chaos theory, fractals, and complexity at the bedside. Lancet, 1996, 347(9011): 1312-1314.

[2] Wolf A, Swift J B, Swinney H L, et al. Determining Lyapunov exponents from a time series. Physical D, 1985, 16: 285-317.

[3] G. barana, I. Tsuda. A new method for computing Lyapunov exponents. Phys. Lett. A, 1993, 175: 421-427.

[4] Zhuping Gong. The Calculation method of chaotic time sequences' average period. System engineering. 2010, 28(12): 111-112.

[5] P. Grassberger and I. Procaccia, Measuring the strangeness of strange attractors. Physical D, 1983, 9: 189-208.

[6] S. M. Pincus. Approximate entropy (ApEn) as a complexity measure, chaos.1995, 5(1): 110-117.

[7] Lempel, J.Ziv. On the Complexity of Finite Sequences. IEEE Trans. Info. Theory, 1976, 22(1): 75-81. 